\title{
Population Dynamics of Insect Pests in Mustard and Eco-friendly Management of Lipaphis erysimi (Kaltenbach) in Uttrakhand
}

\author{
Sundar Pal ${ }^{1 *}$, D.K. Singh ${ }^{2}$ and Ram Singh Umrao ${ }^{3}$
}

${ }^{1}$ DBS College of Agricultur and Allied Science, Selaqui, Dehradun, Uttrakhand-248001, India

${ }^{2}$ Department of Oilseed section, ${ }^{3}$ Department of Entomology, C.S.A.U.A. \& T., Kanpur-208002, India

*Corresponding author

\section{A B S T R A C T}

\section{Keywords}

Mustard aphid,

Sawfly, Painted

bug, Ladybird

beetle and

management

Article Info

Accepted:

04 June 2018

Available Online:

10 July 2018
The peak population of mustard aphid on yellow sticky traps was recorded with $35.4 \pm 2.9$ aphids/trap from $7^{\text {th }} \mathrm{SW}$ and it was active from $45^{\text {th }} \mathrm{SW}$ to $14^{\text {th }} \mathrm{SW}$, where it was on peak with 712.4 \pm 16.4 aphids/plant under the field condition from untreated plots at $3^{\text {rd }} \mathrm{SW}$. Coccinella septempunctatas active from $50^{\text {th }} \mathrm{SW}$ to $13^{\text {th }} \mathrm{SW}$ of the season and peak population noticed from $5^{\text {th }} \mathrm{SW}$ with $14 \pm 0.4$ grubs and adults/plant. Incidence of mustard sawfly was noticed at early stage of crop from $46^{\text {th }} \mathrm{SW}$ to $4^{\text {th }} \mathrm{SW}$ and population range was $0.3 \pm 0.2$ to $7.3 \pm 0.6$ larvae/plant. Painted bug active two times in a season from $45^{\text {th }}-$ $52^{\text {nd }} \mathrm{SW}$ with peak population was $7.5 \pm 0.5$ nymphs and adults/plant and $6^{\text {th }}-12^{\text {th }} \mathrm{SW}$, where peak population was $8.9 \pm 0.5$ nymphs and adults/plant. Imidacloprid $17.8 \%$ SL was most effective against mustard aphid where yield increased $881.38 \mathrm{~kg} / \mathrm{ha}$ and cost benefit ratio was $1: 18.06$ rupees.

\section{Introduction}

Mustard (Brassica jumnica (L.) is an important oilseed crop which is damaged by a number of insect-pests viz., sawfly (Athalia lugens proxima), aphid (Lipaphis erysimi), painted bug (Bagrada cruciferarum) and leaf miner (Phytomyza horticola). Thirty eight insect pests are known to be associated with rapeseed-mustard crop in India (Bakhetia and Sekhon 1989). Among them mustard aphid, Lipaphis erysimi (Kalt.) is the key pest in all the mustard growing regions of the country mustard aphid, L. erysimi (Kaltenbach)
(Homoptera: Aphididae) is the key pest of rape seed mustard crop in India responsible for yield losses ranging from 11.6 to $99 \%$ and oil reduction up to $15 \%$ and also responsible to transmit single-stranded RNA ${ }^{\text {luteo }}$-viruses disease in the crop. In the past, a number of chemical insecticides were evaluated by many workers against this dreaded pest and some of them were effective. These chemical insecticides were found more or less toxic to a number of parasitoids, predators and also to pollinators (Singh and Singh, 2009; Singh et al., 2009). Keot et al., (2002) recorded ten insect pests infesting the brassica vegetables 
right from the seedling stage to the harvest of the crop. The present investigation was undertaken to study the seasonal abundance of aphid on mustard and its eco-friendly management through insecticides and biopesticides.

\section{Materials and Methods}

The field experiment was carried out at DBS College of Agriculture and Allied Science, Dehradun, Uttrakhand, India during 2016-17 and 2017-18. The seeds of local mustard variety sowed in randomized block design (RBD) under three replications (plot size $4.2 \mathrm{x}$ $3 \mathrm{~m} 2$ ) on second week of November 2016 and 2017. Observation recorded on winged aphid, from 10 yellow sticky traps, installed around the experimental field. Chrome painted with smeared transparent greasy material $1.0 \mathrm{~kg}$ oil round tin boxes was installed at ten locations in the experimental farm at $1.5 \mathrm{~m}$ above ground. Population was recorded daily by taking care of cardinal direction throughout the study period. For mustard sawfly, number of larvae/10 plants, for painted bug as number of bugs (adult + nymph)/10 plants and for Coccinella as adult and grub/10 plants at weekly intervals. Observation recorded from treated and untreated plots as the aphid population recorded form top $10 \mathrm{~cm}$ apical twigs of 10 randomly selected from each plot were counted at 3, 7 and 10 days after spray of insecticides, separately. The aphids were removed from the plants with the help of a soft brush and placed on a piece of white paper. Their number was counted with the help of a magnifying glass and tally counter. The numbers of aphids per plant were converted into per cent reduction of aphid population over the control. The observations on damage and mortality were first converted into percentage and then were transformed into angular transformation for statistical analysis to work out the standard error and significant differences among the treatments. The formula used for the calculation of percentage reduction of pest population over control is as follows:

Population reduction over $=100 \quad[1-(\mathrm{Ta} \quad \mathrm{x}$ $\mathrm{Cb}) /(\mathrm{Tb} \times \mathrm{Ca})]$ control $(\%)$

Where,

$\mathrm{Ta}=$ Population in treatment after spray

$\mathrm{Tb}=$ Population in treatment before spray

$\mathrm{Ca}=$ Population in control after spray

$\mathrm{Cb}=$ Population in control before spray.

The meteorological data were also considered during the period of study for correlation calculation.

\section{Results and Discussion}

Population of winged aphid, Lipaphis erysimi attracted on yellow sticky traps

The population of aphid was fluctuating throughout the season. First observation noticed in $45^{\text {th }} \mathrm{WS}$ of the season and numbers of winged aphid attracted on yellow sticky trap was $0.1 \pm 0.1$ aphid/plant. The population was continuing increased to $52^{\text {nd }}$ WS with $13.7 \pm 0.9$ aphids/plant. After that it was decreases on $1^{\text {st }} \mathrm{SW}$ and population was 9.7 \pm 0.4 aphids/plant. This population was again continuing increased to $7^{\text {th }} \mathrm{SW}$ than decreased. The two peak populations of aphid were recorded from $52^{\text {nd }} \mathrm{SW}(13.7 \pm 0.9$ aphids/plant) and second from $7^{\text {th }} \mathrm{SW}$ (35.4 \pm 2.9 aphids/plant) in table 1 . The population was not break in the seasons. The population was positive correlate with $\mathrm{RH}$ but negative with rainfall (\%). Pal et al., (2015) recorded a population of aphid was noticed from last week of December and population was reach in second week of February. Population of winged aphid was very low at the beginning i. e. (0.2 aphid/trap) at the 52nd SW, but attained its maximum density (243.4 aphids/trap) at the 8th SW. Similar peak period was also observed by Kumar et al., (2009). 
Population of aphid, Lipaphis erysimi from plants

The aphid population was noticed from untreated but separated in field from field experiment. The population level was buildup $51.8 \pm 5.9, \quad 244.9 \pm 8.1, \quad 430.6 \pm 8.8, \quad 533.9 \pm 8.0$, $679.9 \pm 14.1$ and $712.4 \pm 16.4$ aphids/plant from $50^{\text {th }}, 51^{\text {st }}, 52^{\text {nd }}, \quad 1^{\text {st }}, \quad 2^{\text {nd }}$ and $3^{\text {rd }}$ WS, respectively. First noticed of aphid population from $48^{\text {th }}$ WS with $1.2 \pm 0.3$ aphids/plant and it was continue increased to $3^{\text {rd }} \mathrm{SW}$ with peak population (712.4 aphids/plant) than it was continue decreased in table 1 . The population was positive correlate with $\mathrm{RH}$ but negative with rainfall (\%). Pal et al., (2015) recorded winged form aphid population from yellow sticky trap 151 aphids/5 traps.

\section{Population of Coccinella septempunctatas}

Predatory population mean of Coccinella was $0.2 \pm 0.2-14.0 \pm 0.4$ grubs and adults/plant. The maximum population of predator was $14.0 \pm 0.4$ grubs and adults/plant from $5^{\text {th }} \mathrm{SW}$ where it was peak point of the season (Table 1). The population of predator was fluctuating according to his prey population in the season. Population of predator was positive correlation with pray population. Singh et al., (2011) was foundund maximum activity of this coccinellid was recorded in the second week of march during 'Rabi' seasons. Bilashini and Singh (2011) observed numerical density of the predator was observed to increase in response to increase in density of aphid prey in the field and the correlation analysis showed highly significant positive correlationship between predator and aphid species.

Population of mustard sawfly, Athalia lugens proxima

Mustard sawfly population was recorded from $46^{\text {th }}$ to $4^{\text {th }} \mathrm{SW}$. Fly population was first time recorded from $46^{\text {th }}$ WS with $2.0 \pm 0.3$ larvae/plant. The population range of mustard sawfly was $0.3 \pm 0.2-7.3 \pm 0.6$ larvae/plant in season (Table 1). The peak population of mustard sawfly was 7.3 larvae/plant recorded from $49^{\text {th }} \mathrm{SW}$.

Pal et al., (2015) recorded mustard sawfly population at early stage where peak population recorded with 2.9 larvae/10 plants. It was non-significant and negative with minimum temperature and $\mathrm{RH}$ but significantly positive with sunshine.

\section{Population of painted bug, Bagrada hilaris}

The population of painted bug was observed two times in a season. First population was recorded from $45^{\text {th }} \mathrm{SW}$ to $52^{\text {nd }} \mathrm{SW}$ and second population observed from $6^{\text {th }} \mathrm{SW}$ to $12^{\text {th }} \mathrm{SW}$. The population range was $0.3 \pm 0.2-7.5 \pm 0.5$ larvae/plant in first population and in second population it was $1.9 \pm 0.2-8.9 \pm 0.5$ larvae/plant. The peak population was $7.5 \pm 0.5$ larvae/plant at $50^{\text {th }} \mathrm{SW}$ in first population and $8.9 \pm 0.5$ larvae/plant at $11^{\text {th }} \mathrm{SW}$ in second population of season in table 1 .

Divya et al., (2015) recorded on incidence of painted bug started in 3 week of December in both years and attained first peak reported during the $1^{\text {st }}$ week of January with 6.75 bugs/plant, whereas, second peak i.e. 7.05 bugs/plant was noticed during the $3^{\text {rd }}$ week of January during 2012-13.

In case of 2013-14, the population of painted bug nd attained a first peak i.e. 6.95 bugs/plant during 2 week of January and second peak i.e. 5.92 bugs/plant rd during 3 week of February. Pal et al.,(2015) recorded the population of painted bug was recorded during 44th to 51st SW and 5th to 11th SW but it was absent from 52nd to 4th SW. The maximum population was recorded 6.1 bugs/10 plants. 
Table.1 Insect pest's incidence in mustard, Brassica species during 2016-17 and 2017-18

\begin{tabular}{|c|c|c|c|c|c|}
\hline \multirow{2}{*}{$\begin{array}{c}\text { Standard } \\
\text { Week }\end{array}$} & \multicolumn{5}{|c|}{ Mean population of insect-pests/10 plants } \\
\cline { 2 - 7 } & $\begin{array}{c}\text { Lipaphis } \\
\text { Meansimi } \\
\text { traps }\end{array}$ & $\begin{array}{c}\text { Untreated } \\
\text { plot }\end{array}$ & $\begin{array}{c}\text { Coccinella } \\
\text { spp. }\end{array}$ & $\begin{array}{c}\text { Athalia lugens } \\
\text { proxima Klug. }\end{array}$ & $\begin{array}{c}\text { Bagrada } \\
\text { hilaris }\end{array}$ \\
\hline 44 & - & - & - & - & - \\
\hline 45 & $0.1 \pm 0.1$ & - & - & - & $0.3 \pm 0.2$ \\
\hline 46 & $0.4 \pm 0.2$ & - & - & $2.0 \pm 0.3$ & $1.1 \pm 0.2$ \\
\hline 47 & $1.7 \pm 0.3$ & - & - & $2.9 \pm 0.4$ & $2.7 \pm 0.3$ \\
\hline 48 & $5.3 \pm 0.4$ & $1.2 \pm 0.3$ & - & $5.0 \pm 0.5$ & $3.2 \pm 0.5$ \\
\hline 49 & $7.0 \pm 0.6$ & $7.4 \pm 3.1$ & - & $7.3 \pm 0.6$ & $5 \pm 0.5$ \\
\hline 50 & $7.4 \pm 0.4$ & $51.8 \pm 5.9$ & $0.2 \pm 0.1$ & $4.6 \pm 0.4$ & $7.5 \pm 0.5$ \\
\hline 51 & $12.1 \pm 0.6$ & $244.9 \pm 8.1$ & $0.4 \pm 0.2$ & $3.6 \pm 0.5$ & $1.3 \pm 0.2$ \\
\hline 52 & $13.7 \pm 0.9$ & $430.6 \pm 8.8$ & $1.1 \pm 0.3$ & $1.8 \pm 0.2$ & $0.3 \pm 0.2$ \\
\hline 01 & $9.7 \pm 0.4$ & $533.9 \pm 8.0$ & $1.2 \pm 0.3$ & $1.8 \pm 0.5$ & - \\
\hline 02 & $9.0 \pm 0.5$ & $679.7 \pm 14.1$ & $6.7 \pm 0.7$ & $0.6 \pm 0.2$ & - \\
\hline 03 & $6.7 \pm 0.6$ & $712.4 \pm 16.4$ & $8.1 \pm 0.7$ & $0.5 \pm 0.2$ & - \\
\hline 04 & $18.7 \pm 1.2$ & $566.5 \pm 10.9$ & $11.9 \pm 0.5$ & $0.3 \pm 0.2$ & - \\
\hline 05 & $18.7 \pm 1.2$ & $436.2 \pm 9.8$ & $14 \pm 0.4$ & - & - \\
\hline 06 & $29.9 \pm 1.4$ & $354.6 \pm 8.8$ & $9.2 \pm 0.4$ & - & $1.9 \pm 0.2$ \\
\hline 07 & $35.4 \pm 2.9$ & $230.6 \pm 5.8$ & $6.6 \pm 0.6$ & - & $2.8 \pm 0.2$ \\
\hline 08 & $15.4 \pm 1.3$ & $192.3 \pm 6.0$ & $4.6 \pm 0.4$ & - & $6.4 \pm 0.3$ \\
\hline 09 & $13.3 \pm 0.7$ & $78.7 \pm 4.0$ & $2.6 \pm 0.5$ & - & $7.0 \pm 0.5$ \\
\hline 10 & $7.7 \pm 0.5$ & $41.3 \pm 4.7$ & $1.3 \pm 0.4$ & - & $8.5 \pm 0.5$ \\
\hline 11 & $3.7 \pm 0.6$ & $23.6 \pm 2.2$ & $0.7 \pm 0.3$ & - & $8.9 \pm 0.5$ \\
\hline 12 & $1.6 \pm 0.5$ & $10 \pm 1.1$ & $0.4 \pm 0.2$ & - & $2.1 \pm 0.2$ \\
\hline 13 & $1.0 \pm 0.3$ & $3.6 \pm 0.6$ & $0.2 \pm 0.2$ & - & - \\
\hline 14 & $0.1 \pm 0.1$ & $0.9 \pm 0.3$ & - & - & - \\
\hline 15 & - & - & - & - & - \\
\hline & & & & & - \\
\hline
\end{tabular}


Table.2 Pooled efficacies of insecticides against mustard aphid (Lipaphis erysimi Kalt.) 2016-17 and 2017-18

\begin{tabular}{|c|c|c|c|c|c|c|c|c|c|c|c|}
\hline \multirow[t]{3}{*}{ Treatments } & \multirow{3}{*}{$\begin{array}{l}\text { Aphid Av. } \\
\text { Population in } \\
\text { number } \\
\text { before spray }\end{array}$} & \multicolumn{10}{|c|}{ Mean aphid population after insecticides application/plants } \\
\hline & & \multicolumn{2}{|c|}{$1^{\text {st }}$ Day } & \multicolumn{2}{|c|}{$3^{\text {rd }}$ Day } & \multicolumn{2}{|c|}{$5^{\text {th }}$ Day } & \multicolumn{2}{|c|}{$7^{\text {th }}$ Day } & \multicolumn{2}{|c|}{$10^{\text {th }}$ Day } \\
\hline & & Population & $\begin{array}{c}\% \\
\text { mortality }\end{array}$ & Population & $\begin{array}{c}\% \\
\text { mortality }\end{array}$ & Population & $\begin{array}{c}\% \\
\text { mortality }\end{array}$ & Population & $\begin{array}{c}\% \\
\text { mortality }\end{array}$ & Population & $\begin{array}{c}\% \\
\text { mortality }\end{array}$ \\
\hline $\begin{array}{c}\text { Acephate } \\
75 \% \text { SP }\end{array}$ & $\begin{array}{l}116.00 \\
(11.27)\end{array}$ & $\begin{array}{c}98.33 \\
(10.42 *)\end{array}$ & $\begin{array}{c}15.23 \\
(22.95 * *)\end{array}$ & $\begin{array}{l}83.33 \\
(9.63)\end{array}$ & $\begin{array}{l}28.16 \\
(32.01)\end{array}$ & $\begin{array}{l}62.33 \\
(8.39)\end{array}$ & $\begin{array}{c}46.27 \\
(42.82)\end{array}$ & $\begin{array}{l}47.67 \\
(7.40)\end{array}$ & $\begin{array}{c}58.91 \\
(50.13)\end{array}$ & $\begin{array}{l}21.67 \\
(5.16)\end{array}$ & $\begin{array}{c}81.32 \\
(64.38)\end{array}$ \\
\hline $\begin{array}{l}\text { Azadirectin } \\
\text { 5SL }\end{array}$ & $\begin{array}{l}120.33 \\
(11.47)\end{array}$ & $\begin{array}{l}110.00 \\
(10.99)\end{array}$ & $\begin{array}{c}8.58 \\
(16.95)\end{array}$ & $\begin{array}{c}90.33 \\
(10.00)\end{array}$ & $\begin{array}{c}23.23 \\
(28.79)\end{array}$ & $\begin{array}{l}78.67 \\
(9.37)\end{array}$ & $\begin{array}{c}34.62 \\
(36.03)\end{array}$ & $\begin{array}{l}57.33 \\
(8.07)\end{array}$ & $\begin{array}{c}52.36 \\
(46.32)\end{array}$ & $\begin{array}{l}28.00 \\
(5.79)\end{array}$ & $\begin{array}{c}76.73 \\
(61.14)\end{array}$ \\
\hline $\begin{array}{c}\text { Carbofuran } \\
3 \mathrm{G}\end{array}$ & $\begin{array}{l}117.67 \\
(11.35)\end{array}$ & $\begin{array}{l}104.00 \\
(10.70)\end{array}$ & $\begin{array}{c}11.62 \\
(19.91)\end{array}$ & $\begin{array}{l}87.33 \\
(9.85)\end{array}$ & $\begin{array}{c}24.28 \\
(29.47)\end{array}$ & $\begin{array}{l}72.67 \\
(9.02)\end{array}$ & $\begin{array}{c}38.24 \\
(38.17)\end{array}$ & $\begin{array}{l}54.00 \\
(7.85)\end{array}$ & $\begin{array}{c}54.11 \\
(47.35)\end{array}$ & $\begin{array}{l}24.00 \\
(5.40)\end{array}$ & $\begin{array}{c}79.60 \\
(63.15)\end{array}$ \\
\hline $\begin{array}{l}\text { Coccinella } \\
\text { Spp. }\end{array}$ & $\begin{array}{l}115.33 \\
(11.24)\end{array}$ & $\begin{array}{l}100.67 \\
(10.53)\end{array}$ & $\begin{array}{c}12.71 \\
(20.88)\end{array}$ & $\begin{array}{c}90.67 \\
(10.02)\end{array}$ & $\begin{array}{c}24.65 \\
(29.73)\end{array}$ & $\begin{array}{l}70.00 \\
(8.87)\end{array}$ & $\begin{array}{c}39.30 \\
(38.82)\end{array}$ & $\begin{array}{l}51.33 \\
(7.66)\end{array}$ & $\begin{array}{c}55.49 \\
(48.10)\end{array}$ & $\begin{array}{l}22.00 \\
(5.19)\end{array}$ & $\begin{array}{c}80.92 \\
(64.08)\end{array}$ \\
\hline $\begin{array}{l}\text { Dimethoate } \\
30 \% \mathrm{EC}\end{array}$ & $\begin{array}{l}112.67 \\
(11.11)\end{array}$ & $\begin{array}{l}94.00 \\
(10.20)\end{array}$ & $\begin{array}{c}16.57 \\
(23.97)\end{array}$ & $\begin{array}{l}76.00 \\
(9.22)\end{array}$ & $\begin{array}{c}32.55 \\
(34.76)\end{array}$ & $\begin{array}{l}59.33 \\
(8.20)\end{array}$ & $\begin{array}{c}47.34 \\
(43.45)\end{array}$ & $\begin{array}{l}38.67 \\
(6.72)\end{array}$ & $\begin{array}{l}65.68 \\
(54.09)\end{array}$ & $\begin{array}{l}18.33 \\
(4.78)\end{array}$ & $\begin{array}{c}83.73 \\
(66.19)\end{array}$ \\
\hline $\begin{array}{c}\text { Imidacloprid } \\
17.8 \% \mathrm{SL}\end{array}$ & $\begin{array}{l}132.33 \\
(12.00)\end{array}$ & $\begin{array}{l}108.00 \\
(10.89)\end{array}$ & $\begin{array}{l}18.39 \\
(25.33)\end{array}$ & $\begin{array}{l}62.67 \\
(8.42)\end{array}$ & $\begin{array}{c}52.64 \\
(46.49)\end{array}$ & $\begin{array}{l}43.33 \\
(7.08)\end{array}$ & $\begin{array}{c}67.26 \\
(55.06)\end{array}$ & $\begin{array}{l}24.00 \\
(5.40)\end{array}$ & $\begin{array}{l}81.86 \\
(64.75)\end{array}$ & $\begin{array}{l}15.00 \\
(4.37)\end{array}$ & $\begin{array}{c}88.66 \\
(70.27)\end{array}$ \\
\hline Control & $\begin{array}{l}128.00 \\
(11.81)\end{array}$ & $\begin{array}{l}124.33 \\
(11.65)\end{array}$ & $\begin{array}{c}2.87 \\
(9.63)\end{array}$ & $\begin{array}{l}123.33 \\
(11.61)\end{array}$ & $\begin{array}{c}3.65 \\
(10.94)\end{array}$ & $\begin{array}{l}121.33 \\
(11.51)\end{array}$ & $\begin{array}{c}5.21 \\
(13.18)\end{array}$ & $\begin{array}{l}123.67 \\
(11.62)\end{array}$ & $\begin{array}{c}3.38 \\
(10.47)\end{array}$ & $\begin{array}{l}125.00 \\
(11.68)\end{array}$ & $\begin{array}{c}2.34 \\
(8.72)\end{array}$ \\
\hline $\mathrm{CD}$ at $5 \%$ tab.vl. & 0.20 & 0.22 & 1.23 & 0.49 & 0.94 & 0.23 & 0.31 & 0.42 & 0.56 & 0.24 & 0.66 \\
\hline
\end{tabular}

*Figure in parentheses $\sqrt{\mathrm{x}}+0.5$ are transferred values. **Figure in parentheses $\sin ^{-1} \sqrt{ }(\mathrm{P} / 100)$ are transferred values.

Table.3 Pooled cost benefit ratio of insecticides against mustard aphid (Lipaphis erysimi Kalt.)

\begin{tabular}{|l|c|c|c|c|c|c|c|c|}
\hline & Dose of & Rate of & Total cost & Yield & Increase & Value of & Net & \\
\hline Acephate 75\%SP & $350 \mathrm{~g} / \mathrm{ha}$ & 350.00 & 1850.00 & 1605.39 & 528.36 & 21134.40 & 19284.40 & $1: 10.42$ \\
\hline Azadirectin 5SL & $5 \mathrm{ml} / \mathrm{L}$ & 1527.00 & 3027.00 & 1512.51 & 435.48 & 17419.20 & 14392.20 & $1: 04.75$ \\
\hline Carbofuran 3G & $12 \mathrm{~g} / \mathrm{plot}$ & 290.15 & 1790.15 & 1544.32 & 467.29 & 18691.60 & 16901.45 & $1: 09.44$ \\
\hline Coccinella Spp. & $5000 \mathrm{grub} / \mathrm{ha}$ & 305.00 & 1805.00 & 1722.22 & 645.19 & 25807.60 & 24002.60 & $1: 13.30$ \\
\hline Dimethoate 30\%EC & $300 \mathrm{~g} / \mathrm{ha}$ & 386.56 & 1886.56 & 1894.05 & 817.02 & 32680.80 & 30794.24 & $1: 16.32$ \\
\hline Imidacloprid 17.8\%SL & $20 \mathrm{~g} / \mathrm{ha}$ & 349.58 & 1849.58 & 1958.41 & 881.38 & 35255.20 & 33405.62 & $1: 18.06$ \\
\hline Control & - & - & - & 1077.03 & - & - & - & - \\
\hline
\end{tabular}

*Labour charges=Rs. 300/labour (four labours)=1200. Rent charges=Rs. 75/machine/day (Two machines and two days)=300. Total cost =Rs. 1500. Marketing price of mustard= Rs. $40.00 / \mathrm{kg}$. 
Plate-1 $^{\text {st }}$
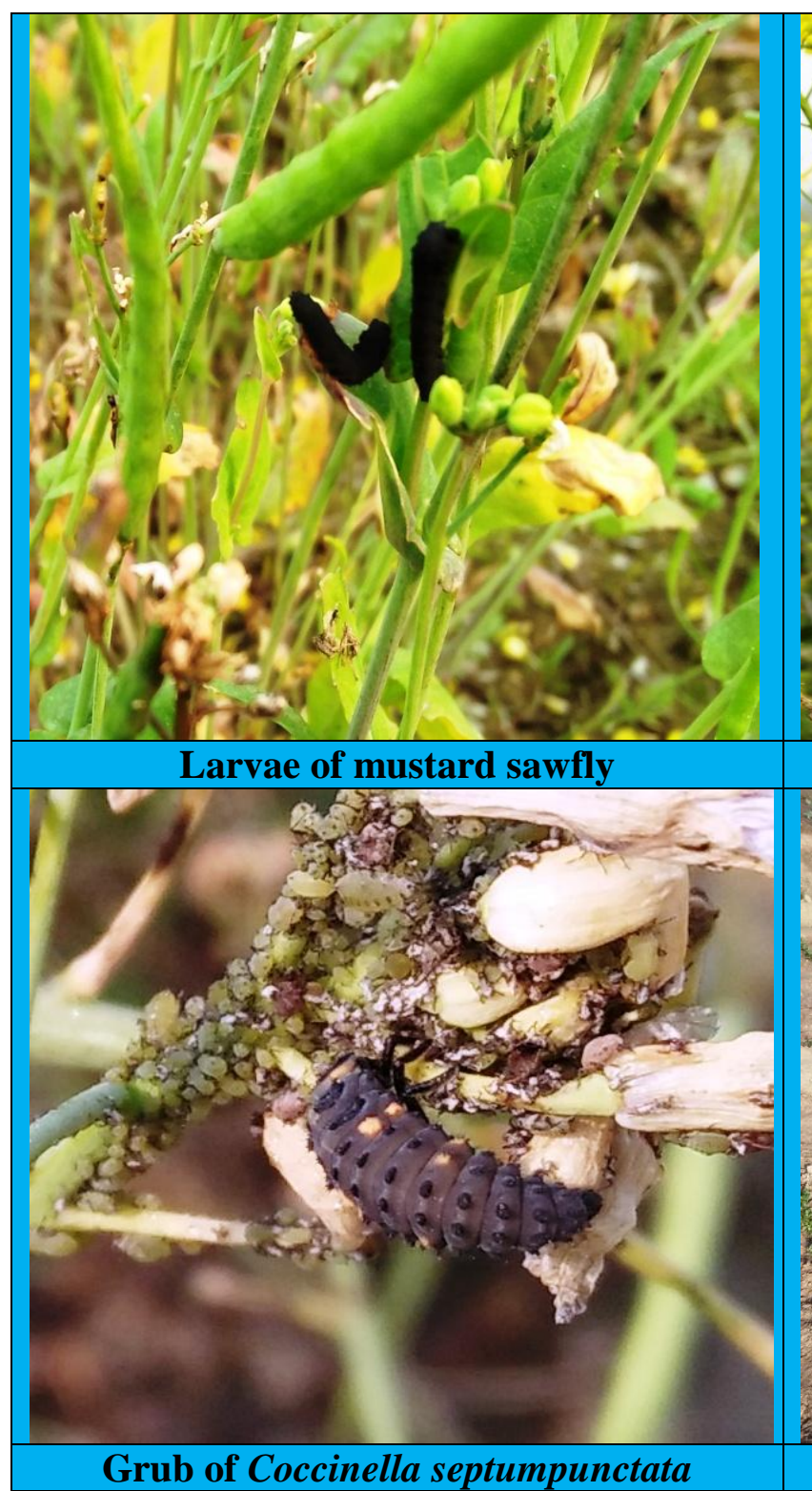

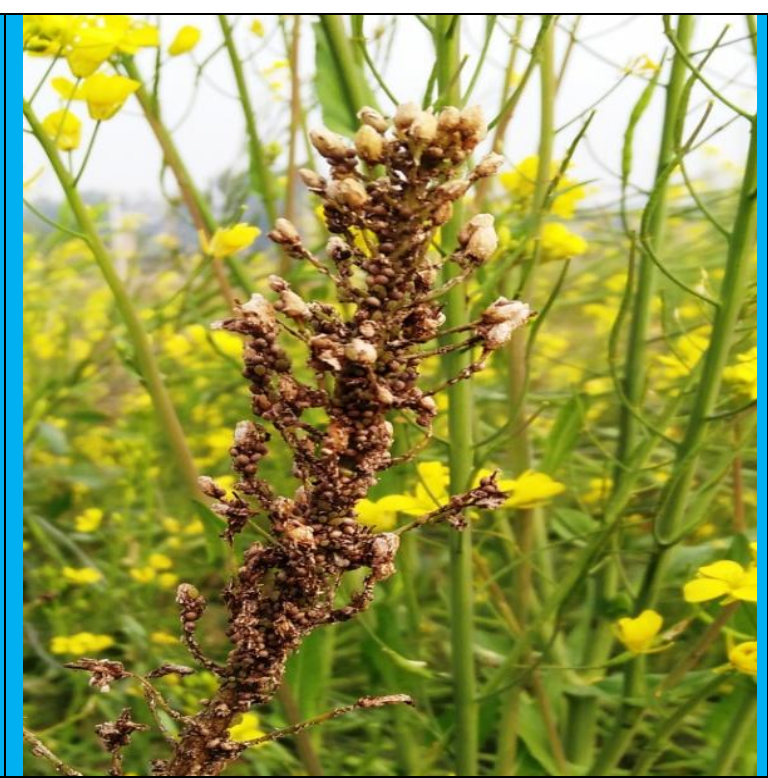

Aphid infectation of mustard plant

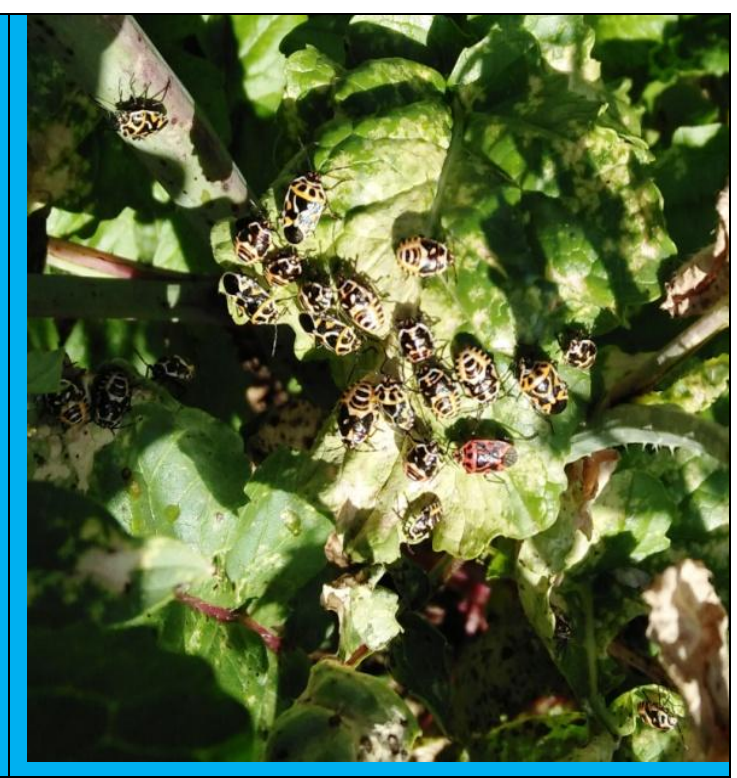

Gregarious form of painted bugs

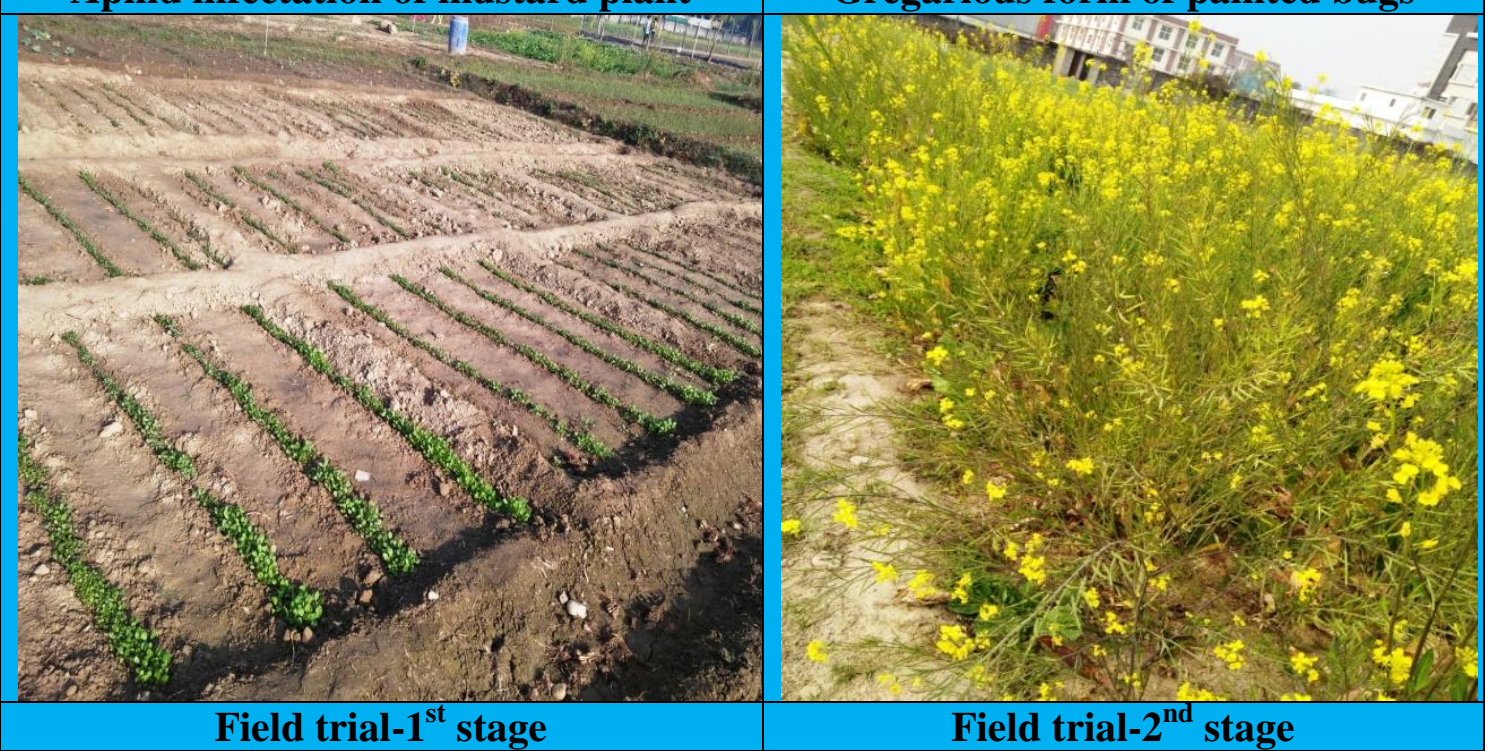


Management of mustard aphid, Lipaphis erysimi

The population of mustard aphid was nonsignificantly difference before spray. Population of aphid was decreased just after spray and data recorded first day after chemicals application. The mortality recorded after chemical application as 94.00, 98.33, 100.67, 104.00, 108.00, 110 and 124.33 aphids/plant followed by before application as $112.67,116.00,115.33$, $117.67,132.33,120.33$ and 128.00 aphids/plant, from dimethoate, acephate, Coccinella septumpunctata, carbofuan, imidacloprid, azadirectin (treated) and control plots, respectively, in table 2 . The mortality per cent was recorded in ascending order, 8.58, 11.62, $12.71, \quad 15.23, \quad 16.57$ and $18.39 \%$ from azadirectin, carbofuan, Coccinella septumpunctata, acephate, dimethoate and imidacloprid, respectively, followed by control. Imidacloprid was best followed by other treatments, but there was no significant difference between carbofuran and Coccinella septumpunctata and acephate and dimethoat. Data recorded on $3^{\text {rd }}$ DAA (Day After Application) the mortality range was 23.2352.64 per cent followed by control. The maximum mortality was recorded from imidacloprid treated plots. The minimum mortality per cent was recorded from azadirectin treated plots. This mortality was superior followed by $1^{\text {st }}$ DAA. There was no significant difference in mortality per cent between azadirectin, carbofuran and Coccinella septumpunctata. Men et al., (2002) recorded seed extract $5 \%$ while dimethoate $0.03 \%$ proved toxic to the parasitoid. Observation was noticed at $5^{\text {th }}$ DAA on mortality per cent in descending order $67.26,47.34,46.27,39.30,38.24$ and 34.62 from imidacloprid, dimethoate, acephate, Coccinella septumpunctata, carbofuan and azadirectin, treated plots, respectively. The population was continue decreased at $7^{\text {th }}$ DAA and observation was recorded 24.00, 38.67, 47.67, 51.33, 54.00 and 57.33 aphids/plant from imidaclopride, dimethoate, acephate, Coccinella septumpunctata, carbofuran and azadirectin treated plots followed by before spray. James
(2003) and Xue (2002) were founded dimethoate and imidacloprid were most toxic against sucking insect pests. The maximum mortality per cent (81.86) was recorded from imodaclopride treated plots followed by control. Boquel et al., (2015) was found most effective 1-4 days after application, contact insecticides strongly modified aphid behavior and intoxicated them. Pal et al., (2015) recorded that the Imidacloprid controlled most effectively followed by oxydemeton methyl, thiamethoxam, dimethoate, fipronil, acephate and acetamiprid. Mortality was continuing at $10^{\text {th }}$ DAA and mortality range was $15.00-28.00$ aphids/plant where mortality per cent range was noticed 76.73-88.66. The effiancy of treatment for mortality per cent was imidacloprid > dimethoate > acephate > Coccinella septumpunctata > carbofuran > azadirectin followed by control.

The efficacy of treatment in mustard was imidacloprid > dimethaote > Coccinella septumpunctata > acephate > carbofuran > azadirectin where net return ₹ $33405.62>$ $30794.24>24002.6>19284.4>16901.45>$ 14392.20, respectively, in table 3. Pal et al., (2015) recorded that the cost : benefit ratio was highest in imidacloprid with a record of maximum monetary benefit of Rs. 29973.36. The cost benefit was ₹ $18.06>16.32>13.30>$ $10.42>9.44>4.75$ after per rupee investment from imidacloprid $>$ dimethaote $>$ Coccinella septumpunctata > acephate > carbofuran > azadirectin, respectively. The maximum yield $(881.38 \mathrm{~kg} / \mathrm{ha})$ was recorded from imidacloprid treated plots. The additional yield of $18.51 \mathrm{q} / \mathrm{ha}$ was also recorded by imidacloprid 17.8 EC $(0.008 \%)$ treated plots over the control (Mishra et al., 2012).

\section{Acknowledgement}

I am highly thankful to the Chairman, DBS College of Agriculture and Allied Science, Selaqui, Dehradun, Uttrakhand for partially supported to carry out the research work. My thankfulness also goes to Principal and other staff members of the college, who provided 
insight and expertise that greatly assisted the research.

\section{References}

Bakhetia DRC Sekhon BS. 1989. Insect pests and their management in rapeseedmustard. J. Oilseeds Res.; 6:269-73.

Bilashini, Y. and Singh, TK 2011. Relative abundance of aphid Lipaphis erysimi (Kaltenbach) and predator Coccinella septempunctata Linnaeus on Brassica oleracea var botrytis in relation with abiotic factors. Environment and Ecology (Kalyani); 29(3A): 1372-1375.

Boquel, Sebastien, Zhang, Jianhua. Goyer, Claudia. Giguere, Marie-Andree. Clark, Catherine. Pelletier, Yvan 2015. Effect of insecticide-treated potato plants on aphid behavior and potato virus $\mathrm{Y}$ acquisition. Pest Management Science., 71(8): 1106-1112.

Divya, C., R. Kalsariya and HG Kanara 2015. Seasonal incidence of mustard painted bug, Bagrada hilaris (Burmeister) and their correlation with abiotic factors on mustard. Conference: 4th Congress on Insect Science, At PAU, Ludhiana, Volume: 28(1).

James, D.G., 2003. Pesticide Susceptibility of Two Coccinellids (Stethorus punctum picipes and Harmonia axyridis) Important in Biological Control of Mites and Aphids in Washington Hops. Biocontrol Science and Technology. 13(2):253-259.

Keot, S. Saikia, DK. Nath, RK., 2002. Insect pest complex of Brassica vegetables. JASS; 15(1): 28-33.

Kumar, Gaurav, Anandhi, P. Varma, Savita and
Elamathi, S. 2009. Seasonal occurrence of Brevicoryne brassicae and natural enemies on cabbage. Ann. Pl. Protec.Sci. 17 : 476-478.

Men, UB. Bhabad, NS. Kandalkar, HG 2002. Relative safety of some biopesticides and insecticides to Diaeretiella rapae (Mc'Intosh), a parasitoid of mustard aphid, Lipaphis erysimi (Kaltenbach). Pest Management and Economic Zoology;10(2): 201-203.

Mishra, M. K., Singh, R. P. and Ali, S. 2012. Chemical control and avoidable yield losses of pigeonpea due to insect-pests. Ann. Pl. Protec. Sci. 20 : 306-309.

Pal, Sundar, D. K. Singh, Sanjeev Kumar, C. S. Shekhawat and Dheeraj Kumar 2015. Seasonal Abundance of Insect-pests on Mustard Varieties (Brassica spp.) and Relation to Abiotic Factors. Annals of Biology, 31 (1) : 109-112.

Pal, S., D. K. Singh, D. N. Singh, R. S. Umrao and R. K. Dwivedi 2015. Monitoring and Management of Mustard Aphid, Lipaphis erysimi Kalt. Annals of AgriBio Research, 20 (1) : 26-29.

Singh, S. P. and Singh, Y. P. 2009. Bio-efficacy of pesticides against mustard aphid. Ann. Pl, Protec. Sci. 17 : 240-242.

Singh, S. P., Singh Y. P., Singh Ram and Nagar, Rajender 2009. Efficacy of botanicals in the management of mustard aphid, Lipaphis erysimi Kalt. and their impact on beneficial insects. $J$. of Oilseeds Res. 29: 113-115.

Xue, Ming. Li, Qiang 2002. Studies on selective toxicity of six insecticides between green peach aphid and ladybirds. Entomologia Sinica; 9(2): 17-22.

\section{How to cite this article:}

Sundar Pal, D.K. Singh and Ram Singh Umrao. 2018. Population Dynamics of Insect Pests in Mustard and Eco-friendly Management of Lipaphis erysimi (Kaltenbach) in Uttrakhand. Int.J.Curr.Microbiol.App.Sci. 7(07): 324-331. doi: https://doi.org/10.20546/ijcmas.2018.707.039 